\title{
An Extension of a Theorem of Yao and Yao
}

\author{
Edgardo Roldán-Pensado • Pablo Soberón
}

Received: 8 April 2012 / Revised: 23 October 2013 / Accepted: 6 January 2014 /

Published online: 16 January 2014

(C) Springer Science+Business Media New York 2014

\begin{abstract}
In this paper we study $N_{d}(k)$, the smallest positive integer such that any nice measure $\mu$ in $\mathbb{R}^{d}$ can be partitioned into $N_{d}(k)$ convex parts of equal measure so that every hyperplane avoids at least $k$ of them. A theorem of Yao and Yao states that $N_{d}(1) \leq 2^{d}$. Among other results, we obtain the bounds $N_{d}(2) \leq 3 \cdot 2^{d-1}$ and $N_{d}(1) \geq C \cdot 2^{d / 2}$ for some constant $C$. We then apply these results to a problem on the separation of points and hyperplanes.
\end{abstract}

Keywords Convex partition · Measure equipartition · Yao-Yao theorem

\section{Introduction}

A convex partition of $\mathbb{R}^{d}$ into $n$ parts is a covering $\mathcal{P}=\left\{C_{1}, \ldots, C_{n}\right\}$ of $\mathbb{R}^{d}$ consisting of closed convex bodies with pairwise disjoint interiors. We say that a hyperplane $H \subset \mathbb{R}^{d}$ avoids a set $C$ if it does not intersect its interior.

The classical Yao-Yao theorem [12] states the following.

Theorem (Yao and Yao, 1985) Let $\mu$ be a nice measure in $\mathbb{R}^{d}$, then there is a convex partition $\mathcal{P}$ of $\mathbb{R}^{d}$ into $2^{d}$ parts of equal $\mu$-measure such that every hyperplane in $\mathbb{R}^{d}$ avoids at least one element of $\mathcal{P}$.

In the original proof of this theorem the measure has to have a continuous density function bounded away from $O$. Later, in [7], this was weakened to the condition that the measure of every hyperplane be 0 . In this paper we ask $\mu$ to satisfy the original

E. Roldán-Pensado · P. Soberón

Mathematics Department, University College London, London WC1E 6BT, UK

e-mail: pablo.soberon@ciencias.unam.mx

E. Roldán-Pensado

e-mail: e.roldan@math.ucl.ac.uk 
Table 1 Upper bounds for $N_{2}$

\begin{tabular}{llllllll}
\hline$k$ & $N_{2}(k)$ & $k$ & $N_{2}(k)$ & $k$ & $N_{2}(k)$ & $k$ & $N_{2}(k)$ \\
\hline 1 & 4 & 6 & 14 & 11 & 24 & 16 & 32 \\
2 & 6 & 7 & 16 & 12 & 24 & 17 & 32 \\
3 & 8 & 8 & 18 & 13 & 28 & 18 & 36 \\
4 & 10 & 9 & 20 & 14 & 30 & 19 & 36 \\
5 & 12 & 10 & 22 & 15 & 32 & 20 & 36 \\
\hline
\end{tabular}

conditions, as in [12]. We say that a measure that satisfies these conditions is a nice measure

This theorem gives a partition in which almost every hyperplane intersects exactly $2^{d}-1$ pieces. The proof gives a unique partition for each ordered orthonormal basis $\left(u_{1}, \ldots, u_{d}\right)$. We extend this theorem to the case when every hyperplane is required to avoid 2 pieces.

Theorem 1 Let $\mu$ be a nice measure in $\mathbb{R}^{d}$, then there is a convex partition $\mathcal{P}$ of $\mathbb{R}^{d}$ into $3 \cdot 2^{d-1}$ parts of equal $\mu$-measure such that every hyperplane in $\mathbb{R}^{d}$ avoids at least two elements of $\mathcal{P}$.

For $d=2$ this follows from a theorem by Buck and Buck [4] which states that any nice measure $\mu$ in $\mathbb{R}^{2}$ can be divided into six parts of equal measure by three concurrent lines. Our method gives in this case a partition by three lines, two of which are parallel. The proof of Theorem 1 is given in Sect. 4. Some details of Yao and Yao's original proof are necessary, so a sketch of their methods is given in Sect. 3.

Let $N_{d}(k)$ be the smallest positive integer such that the following holds: For every nice measure $\mu$ on $\mathbb{R}^{d}$ there exists a partition of $\mathbb{R}^{d}$ into $N_{d}(k)$ convex parts of equal measure such that every hyperplane avoids at least $k$ parts. We call such a partition a $k$-equipartition. Yao-Yao's theorem and Theorem 1 are equivalent to the bounds $N_{d}(1) \leq 2^{d}$ and $N_{d}(2) \leq 3 \cdot 2^{d-1}$.

There is another number which seems useful. Let $M_{d}(k, \alpha)$ be the smallest positive integer such that for every nice measure $\mu$ on $\mathbb{R}^{d}$ there is a family of $M_{d}(k, \alpha)$ convex sets such that every hyperplane avoids at least $k$ of them and they all have measure at least $\alpha$. Clearly we have

$$
N_{d}(k) \geq M_{d}\left(k, \frac{1}{N_{d}(k)}\right)
$$

For $N_{d}$ there is a simple bound in dimension 2 that strictly improves the bounds that can be obtained by using Yao-Yao's theorem, Theorem 1 and the formulas of Sect. 5 for all $k \leq 15$ except $k=1,2,7,12$ (see Table 1).

Proposition $2 N_{2}(k) \leq 2 k+2$.

For a fixed $d$, we can determine the asymptotic behavior of $N_{d}(k)$.

Theorem $3 \lim _{k \rightarrow \infty} \frac{N_{d}(k)}{k}=1$. 
This means that the condition of equipartitioning a given measure is not very strong in the sense that, if there are enough parts, it can be done so that every hyperplane avoids almost all of them. This is the same behavior as one would expect from a random partition. These results are proven in Sect. 5. In Sect. 2 they are applied to an apparently simple but annoyingly resistant problem regarding separation of points and hyperplanes.

Problem Determine all pairs $(\alpha, \beta) \in \mathbb{R}_{+}^{2}$ such that for any finite set $X$ of points in $\mathbb{R}^{d}$ and any finite set $Y$ of hyperplanes in $\mathbb{R}^{d}$, there are sets $A \subset X$ and $B \subset Y$ such that:

- $|A| \geq \alpha|X|$,

- $|B| \geq \beta|Y|$,

- no two points in $A$ are separated by a hyperplane in $B$.

One should note that this problem is similar to Theorem 18 in [5], and the proof method used for that problem also involves the Yao-Yao theorem. Trying to solve the question above we obtained a bound for $N_{d}(1)$ in terms of $h_{d}(t)$, the measure of a spherical cap of $S^{d}$ with central angle $t$. This measure is computed with the usual probability measure in $S^{d}$.

Theorem 4 Let $\alpha>0$ be such that there is a family A of convex sets in $\mathbb{R}^{d}$ with the following properties:

- Every set in A has measure at most $\alpha$,

- every hyperplane avoids at least one set of $A$,

- the sum of the measures of the sets in A is not greater than 1.

Then

$$
\alpha \leq 2 \cdot h_{d}\left(\frac{\pi}{4}\right) \leq 2 \cdot 2^{-\frac{d}{2}} .
$$

The hypotheses of this theorem can be written simply as $1 \geq \alpha \cdot M_{d}(1, \alpha)$. Note that if $A$ is a 1 -equipartition, the conditions above hold. Thus, we can set $\alpha=\frac{1}{N_{d}(1)}$. This is also implied by (1).

As a consequence we obtain,

\section{Corollary 5}

$$
N_{d}(1) \geq 2^{\frac{d}{2}-1}
$$

For the approximation of the cap measure see [2], for example. Thus, an exponentially large number of parts is needed. This answers a question by B. Bukh on whether the number of pieces needed is indeed super-polynomial. This kind of bound can be obtained for $N_{d}(k)$ for any $k$ in terms of spherical caps of $S^{d}$. However, explicit approximations are hard to find. These results are a consequence of Proposition 6 and Theorem 9 below. 
Remark The Yao-Yao theorem can be generalized in the following way: Given $a_{1}, a_{2}, \ldots, a_{2^{d}}>0$ such that $\sum a_{i}=\mu\left(\mathbb{R}^{d}\right)$, there is a partition of $\mathbb{R}^{d}$ into $2^{d}$ convex parts $\left\{C_{1}, C_{2}, \ldots, C_{2^{d}}\right\}$ such that $\mu\left(C_{i}\right)=a_{i}$ for all $i$ and every hyperplane avoids the interior of at least one $C_{i}$. Theorem 1 can also be generalized in the same way. This is made clear in the next sections.

\section{The $(\alpha, \beta)$ Problem}

The $(\alpha, \beta)$ problem deals with how well behaved points and hyperplanes are with each other in terms of separation. This problem was told to the authors by I. Bárány in its first version, mentioned in Sect. 1. It has the difficulty that it is not self-dual, so we work with a second version which does have this property.

Problem (Second version) Find all pairs $(\alpha, \beta) \in \mathbb{R}_{+}^{2}$ such that for any two nice centrally symmetric probability measures $\mu_{1}, \mu_{2}$ in $S^{d}$ there are sets $A, B \subset S^{d}$ with $\mu_{1}(A) \geq \alpha, \mu_{2}(B) \geq \beta$ such that either

$$
\begin{array}{ll}
a \cdot b \geq 0 & \text { for all } a \in A, b \in B \quad \text { or } \\
a \cdot b \leq 0 & \text { for all } a \in A, b \in B .
\end{array}
$$

Here the condition of the measures being centrally symmetric is not really needed. If they are not then we can consider the measures given by $\mu_{1}^{\prime}(A)=\frac{1}{2}\left(\mu_{1}(A)+\right.$ $\left.\mu_{1}(-A)\right)$ and $\mu_{2}^{\prime}(A)=\frac{1}{2}\left(\mu_{2}(A)+\mu_{2}(-A)\right)$ and obtain the same pairs $(\alpha, \beta)$ for these measures.

Let $\mathcal{C}_{d}^{\prime}$ be the set of pairs $(\alpha, \beta)$ that satisfy the conditions of the original problem and $\mathcal{C}_{d}$ be the set of pairs that satisfy the conditions of the second version of the problem.

Note that $(\alpha, \beta) \in \mathcal{C}_{d}^{\prime}$ if and only if $\left(\frac{\alpha}{2}, \frac{\beta}{2}\right) \in \mathcal{C}_{d}$. This is done simply by embed$\operatorname{ding} \mathbb{R}^{d}$ in $\mathbb{R}^{d+1}$ as a hyperplane not containing the origin. Then every point $a \in \mathbb{R}^{d}$ corresponds to the pair of points $\left\{a^{\prime},-a^{\prime}\right\}$ in $S^{d}$ in the line $O a$ and every hyperplane $H \in \mathbb{R}^{d}$ corresponds to the pair of points $\left\{b^{\prime},-b^{\prime}\right\}$ in $S^{d}$ such that $b^{\prime}$ is orthogonal to every line $O b$ with $b \in H$.

With this transformation we see that the original problem is essentially equivalent to one similar to the second version but with symmetric finite sets of points instead of probability measures. The change to measures follows from the fact that every nice measure can be approximated by linear combinations of Dirac measures and vice versa.

The sets $A$ and $B$ in this problem can (and will) be taken as the intersection of $S^{d}$ and a convex cone in $\mathbb{R}^{d+1}$ with apex at the origin.

We shall denote by $M^{d}$ the usual probability measure on $S^{d}$. Given $0 \leq t<\pi$ and $x \in S^{d}$, let $C(d, x, t)$ be the spherical cap of $S^{d}$ with center $x$ and central angle $t$. Denote by $h_{d}(t)$ its $M^{d}$-measure.

Given $A \subset S^{d}$, let $A^{\top}$ be the set of points $x \in S^{d}$ such that there exists $a \in A$ with $a \cdot x=0$. Note that if $A$ is connected, the largest set $B \subset S^{d}$ that satisfies (2) is one of the connected components of the complement of $A^{\top}$. 
Given a set $A$ of fixed measure, in order to bound the measure of $B$ we need a variant of Lévy's isoperimetric inequality [8]. With our notation, Theorem 2.1 in [6] states the following.

Theorem Let $A$ be a closed subset of $S^{d}$ and set $t>0$ so that $M^{d}(A)=h_{d}(t)$. Then for every $\varepsilon>0, M^{d}\left(A_{\varepsilon}\right) \geq h_{d}(t+\varepsilon)$, where $A_{\varepsilon}$ is the set of points $x \in S^{d}$ with geodesic distance smaller than $\varepsilon$ from $x$ (i.e. the set of points for which there exists $a \in A$ with $\arccos (a \cdot x)<\varepsilon)$.

If $\varepsilon=\frac{\pi}{2}$ and $A$ is connected, then $S^{d} \backslash A_{\varepsilon}$ is one of the two connected components of $S^{d} \backslash A^{\top}$. Therefore, if $\mu_{1}$ and $\mu_{2}$ are equal to $M^{d}$ and $A, B \subset S^{d}$ satisfy (2) and $M^{d}(A)=h_{d}(t)$ for some $t>0$, then $M^{d}(B) \leq h_{d}\left(\frac{\pi}{2}-t\right)$. This is the following proposition.

Proposition 6 All points in $\mathcal{C}_{d}$ lie on or below the curve

$$
\left\{\left(h_{d}(t), h_{d}\left(\frac{\pi}{2}-t\right)\right): 0 \leq t \leq \frac{\pi}{2}\right\} .
$$

This turns out to be best possible if $d=1$.

\section{Theorem 7}

$$
\mathcal{C}_{1}=\left\{(\alpha, \beta): \alpha+\beta \leq \frac{1}{2}\right\}
$$

Proof Take $\alpha, \beta$ with $\alpha+\beta \leq \frac{1}{2}$. Suppose that for every arc segment $A$ with $\mu_{1}(A)=$ $\alpha$ we have $\mu_{2}\left(A^{\top}\right)>2 \alpha$. Let $\phi$ be the rotation of $S^{1}$ by an angle of $\frac{\pi}{2}$ and note that $A^{\top}=\phi(A) \cup \phi^{-1}(A)$. Since $\mu_{1}$ and $\mu_{2}$ are centrally symmetric then $\phi(A)=$ $\phi^{-1}(A)$ and therefore $\mu_{1}(A)<\mu_{2}(\phi(A))$. Since this happens for every arc $A$ with $\mu_{1}$-measure $\alpha, \mu_{1}\left(S^{1}\right)<\mu_{2}\left(S^{1}\right)$, which is a contradiction. Therefore there exists an arc segment $A$ that satisfies $\mu_{2}\left(A^{\top}\right) \leq 2 \alpha$. If $B$ is any of the two components of $S^{1} \backslash A^{\top}$, then $\mu_{2}(B) \geq \beta$. This proves one of the inclusions, Proposition 6 gives the other.

The Yao-Yao type partition theorems can be used to find pairs in the $(\alpha, \beta)$ problem. The following lemma is the main tool for this purpose.

Lemma 8 Let $0 \leq \rho \leq 1$. Suppose that for any nice measure $\mu_{1}$ on $S_{d}$ there exists a family $F$ of closed connected subsets of $S^{d}$ and a probability measure $\mu_{F}$ on $F$ such that

- $\mu_{1}(A) \geq \alpha$ for all $A \in F$,

- For every $b \in S^{d}$, the set $F_{b}=\left\{A \in F: A \cap\{b\}^{\top} \neq \emptyset\right\}$ is $\mu_{F}$-measurable and $\mu_{F}\left(F_{b}\right) \leq \rho$.

Then $\left(\alpha, \frac{1-\rho}{2}\right) \in \mathcal{C}_{d}$. 
Proof Let $\mu_{1}$ and $\mu_{2}$ be nice measures. Suppose that we can find $F$ and $\mu_{F}$ for $\mu_{1}$ as above, then by Fubini's Theorem

$$
\begin{aligned}
\int_{F} \mu_{2}\left(A^{\top}\right) d \mu_{F} & =\int_{F} \int_{S^{d}} \chi\left(A^{\top}\right) d \mu_{2} d \mu_{F}=\int_{S^{d}} \int_{F} \chi\left(F_{b}\right) d \mu_{F} d \mu_{2} \\
& =\int_{S^{d}} \mu_{F}\left(F_{b}\right) d \mu_{2} \leq \rho,
\end{aligned}
$$

where $\chi$ is the characteristic function of a set. Thus, we can find $A_{0}$ such that $\mu_{2}\left(A_{0}^{\top}\right) \leq \rho$. This means that there is a set $B$ such that $\mu_{1}(B) \geq \frac{1-\rho}{2}$ and the sign of $a \cdot b$ is constant for all $a \in A_{0}$ and $b \in B$.

Using this with $N_{d}(k)$ and $M_{d}(k, \alpha)$, we obtain the following.

Theorem 9 For any two positive integers $k$ and $d$,

$$
\left(\frac{1}{2 N_{d}(k)}, \frac{k}{2 N_{d}(k)}\right) \in \mathcal{C}_{d}
$$

More generally, if $\alpha>0$,

$$
\left(\frac{\alpha}{2}, \frac{k}{2 M_{d}(k, \alpha)}\right) \in \mathcal{C}_{d}
$$

Proof Given a hyperplane in $\mathbb{R}^{d+1}$ that does not pass through the origin, consider the radial projection $\mathbb{R}^{d} \rightarrow S^{d}$. Since the measure of every great circle in $S^{d}$ has null $\mu$-measure, this induces a probability measure $\mu^{\top}$ in $\mathbb{R}^{d}$. Fix a family of $M_{d}(k, \alpha)$ convex sets of measure $\alpha$ such that every hyperplane (in $\mathbb{R}^{d}$ ) avoids the interior of at least $k$ of them. If we bring this family back to $S^{d}$, we obtain a new family $\mathcal{F}$ of $2 M_{d}(k, \alpha)$ convex sets in $S^{d}$, each of measure $\frac{\alpha}{2}$. By choosing $\mu_{F}$ to be the uniform probability measure on $F$ and applying Lemma 8, we are done.

We are now ready to prove Theorem 4 .

Proof of Theorem 4 Note that the conditions of Theorem 4 can be written as $\frac{1}{M_{d}(1, \alpha)} \geq \alpha$. Thus, since $\left(\frac{\alpha}{2}, \frac{1}{2 M_{d}(1, \alpha)}\right) \in \mathcal{C}_{d}$ by Theorem 9 , we have $\left(\frac{\alpha}{2}, \frac{\alpha}{2}\right) \in \mathcal{C}_{d}$. Finally, by Proposition 6 we obtain $\frac{\alpha}{2} \leq h_{d}\left(\frac{\pi}{4}\right)$, as we wanted.

It should be noted that the same method also implies lower bounds for $N_{d}(k)$ for any $k$.

Applying the results obtained in Sect. 5 for $N_{d}(k)$, we can show the following.

Corollary 10 For any two non-negative integers $k_{1}$ and $k_{2}$, not both equal to 0 , we have

$$
\frac{1}{2}\left(\left(\frac{1}{2^{d}}\right)^{k_{1}}\left(\frac{1}{3 \cdot 2^{d-1}}\right)^{k_{2}}, 1-\left(1-\frac{1}{2^{d}}\right)^{k_{1}}\left(1-\frac{1}{3 \cdot 2^{d-2}}\right)^{k_{2}}\right) \in \mathcal{C}_{d}
$$



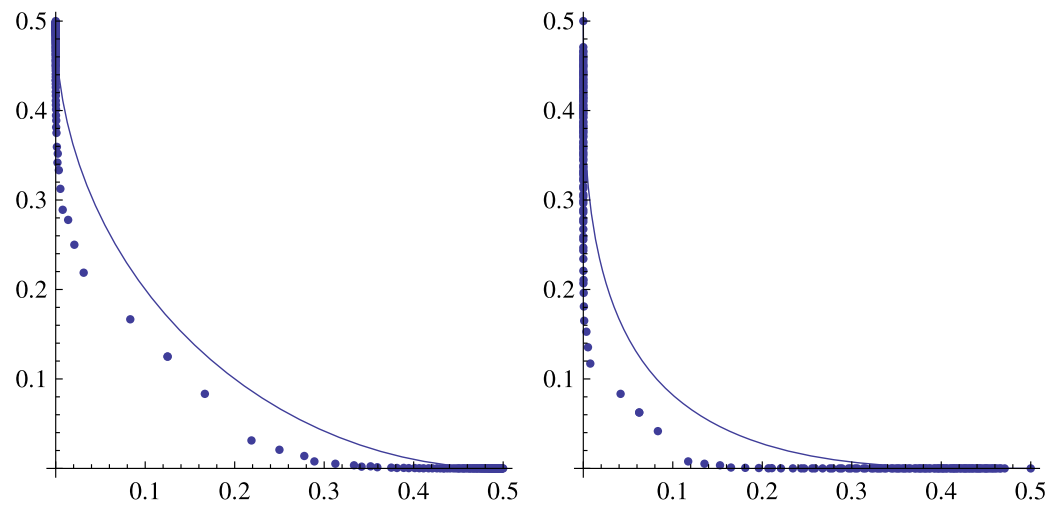

Fig. 1 Bounds for $\mathcal{C}_{2}$ and $\mathcal{C}_{3}$

This gives in particular that $\left(\frac{1}{2^{d+1}}, \frac{1}{2^{d+1}}\right) \in \mathcal{C}_{d}$ and $\left(\frac{1}{3 \cdot 2^{d}}, \frac{1}{3 \cdot 2^{d-1}}\right) \in \mathcal{C}_{d}$. The fact that $\left(\frac{1}{2^{d+1}}, \frac{1}{2^{d+1}}\right) \in \mathcal{C}_{d}$ was obtained earlier in [1] using a similar method. In Fig. 1 there are plots of these points together with the bound obtained in Proposition 6 in dimensions 2 and 3 .

Corollary 11 There are pairs $(\alpha, \beta) \in \mathcal{C}_{d}$ arbitrarily close to $\left(0, \frac{1}{2}\right)$.

This last corollary comes from the fact that $\lim N_{d}(k) / k=1$. However, as these pairs get close to $\left(0, \frac{1}{2}\right)$ they are significantly worse than what Proposition 6 gives.

We can get better bounds if further conditions are imposed on one of the measures. Let $\mathcal{C}_{d}(\Delta)$ be the set of pairs $(\alpha, \beta)$ such that for any two measures $\mu_{1}, \mu_{2}$ on $S^{d}$ such that $\mu_{1}$ has density $f$ with respect to $M^{d}$ satisfying $\operatorname{Lip}(f) \leq \Delta$, there are sets $A, B$ in $S^{d}$ satisfying $\mu_{1}(A)=\alpha, \mu_{2}(B)=\beta$ and either $a \cdot b \geq 0$ for all $a \in A, b \in B$ or $a \cdot b \leq 0$ for all $a \in B, b \in B$.

Theorem 12 For every $0<\lambda \leq 1$ and $0<r<\frac{1-\lambda}{\Delta}$ we have

$$
\left(\lambda h_{d}(r), h_{d-1}\left(\frac{\pi}{2}-2 \arcsin \left(\frac{\sin (r)}{\sin \left(\frac{1-\lambda}{\Delta}-r\right)}\right)\right)\right) \in \mathcal{C}_{d}(\Delta) .
$$

If $r$ is close to 0 , then the pairs obtained are close to

$$
\left(\lambda h_{d}(r), h_{d-1}\left(\frac{\pi}{2}-c_{2} r\right)\right)
$$

for a constant $c_{2}$ depending on $\lambda$ and $\Delta$. That is, the difference in dimension with respect to the bounds of Proposition 6 is compensated by the constants. The idea of the proof is to use a small $S^{d-1}$ in $S^{d}$ and the Lipschitz condition to construct sets as in the proof of Lemma 8. If instead of this we use a hypercube (of dimension $d-1$ ) in $S^{d}$, we obtain bounds of the type $\left(\frac{c_{1}}{m^{d-1}}, \frac{1}{2}-\frac{c_{2} d}{m}\right)$. These are worse than the ones in Theorem 12 but are easier to grasp. 
Fig. 2 The family for Theorem 12

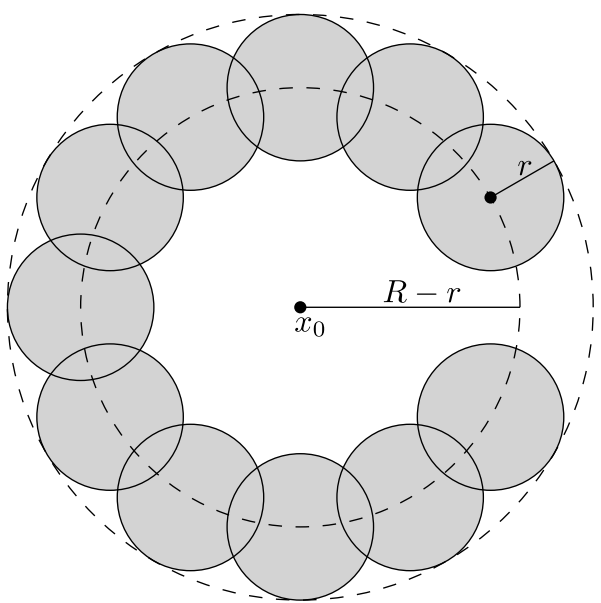

Proof of Theorem 12 Since $\mu_{1}\left(S^{d}\right)=1$, there must be a point $x_{0} \in S^{d}$ such that $f\left(x_{0}\right) \geq 1$. Consider $R=\min \left(\frac{1-\lambda}{\Delta}, \frac{\pi}{2}\right)$. Since $\operatorname{Lip}(f) \leq \Delta$, it follows that $f(x) \geq \lambda$ for all $x \in S^{d}$ at distance at most $R$ from $x_{0}$. Consider $r \leq \frac{R}{4}$. Given two points $x, y \in S^{d}$, denote by $\operatorname{dist}_{S^{d}}(x, y)$ their distance in the sphere. Namely, the angle they sustain at the origin. For each $x \in S^{d}$, define

$$
S(x, r)=\left\{y \in S^{d}: \operatorname{dist}_{S^{d}}(x, y) \leq r\right\} .
$$

We construct the family $F$ as follows (see Fig. 2):

$$
F=\left\{S(x, r): \operatorname{dist}\left(x, x_{0}\right)=R-r\right\} .
$$

We can note that

- Each set in $F$ has measure at least $\lambda h_{d}(r)$

- $S(x, r)$ is the intersection of $S^{d}$ with a ball with center $x$ and radius $\sin (r)$.

- The locus of the centers of the balls in $F$ is $S\left(x_{0}, R-r\right)$; the intersection of $S^{d}$ with a ball with center $x_{0}$ and radius $\sin (R-r)$.

Consider $\mu_{F}$ the usual probability measure on $S\left(x_{0}, R-r\right)$. Then, every hyperplane through the center of $S^{d}$ intersects a subset of $F$ of size at most

$$
1-2 h_{d-1}\left(\frac{\pi}{2}-2 \arcsin \left(\frac{\sin (r)}{\sin (R-r)}\right)\right) \text {. }
$$

Then, the same argument for Lemma 8 finishes the proof.

\section{Yao-Yao's Original Proof}

We give a sketch of the original proof by Yao and Yao since its spirit will be followed in the proof of our main theorem. This will also allow us to note some additional properties and fix notation. 
Let $O(d)$ be the space consisting of $d \times d$ matrices $u$ such that $u^{T} u=I$, the space $S O(d) \subset O(d)$ consists of matrices with determinant 1. A matrix $u \in O(d)$ can be expressed as $u=\left(u_{1}, \ldots, u_{d}\right)$ where $u_{i}$ is the $i$ th row vector of $u$. In this way every $u$ can be identified with an ordered orthonormal base of $\mathbb{R}^{d}$.

Fix a base $u=\left(u_{1}, \ldots, u_{d}\right)$ of $\mathbb{R}^{d}$. If $H$ is a hyperplane orthogonal to $u_{1}$, define the open half-spaces

$$
\begin{aligned}
& H^{+}=\left\{x+t u_{1}: x \in H, t>0\right\}, \\
& H^{-}=\left\{x-t u_{1}: x \in H, t>0\right\} .
\end{aligned}
$$

Let $v$ be a unit vector in $\mathbb{R}^{d}$ not orthogonal to $u_{1}$ and let $p_{v}: \mathbb{R}^{d} \rightarrow H$ be the projection such that $p_{v}(x+t v)=x$ for all $x \in H$ and $t \in \mathbb{R}$. We can identify $H$ with $\mathbb{R}^{d-1}$ by means of the base $u_{2}, \ldots, u_{d}$. There is a natural way to define measures $\mu_{v}^{+}$ and $\mu_{v}^{-}$in $H$ : For any measurable $S \subset H$, set $\mu_{v}^{ \pm}(S)=\mu\left(p_{v}^{-1}(S) \cap H^{ \pm}\right)$.

In [12] a center $c \in \mathbb{R}^{d}$ for $\mu$ relative to the base $u_{1}, \ldots, u_{d}$ is defined as follows:

- If $d=1$ then $c$ is the point that splits $\mathbb{R}$ into two parts of equal $\mu$-measure.

- If $d>1$, let $H$ be the hyperplane orthogonal to $u_{1}$ that splits $\mathbb{R}^{d}$ into two parts of equal $\mu$-measure. Then $c$ lies on $H$ and there exists a unit vector $v\left(\right.$ with $u_{1} \cdot v>0$ ) such that $c$ is a center for both $\mu_{v}^{+}$and $\mu_{v}^{-}$relative to $u_{2}, \ldots, u_{d}$.

Then it is proved that $c$ exists and is unique. Inductively it is easy to see that this induces a partition of $\mathbb{R}^{d}$ into $2^{d}$ parts and each part is a cone bounded by $d$ hyperplanes having $c$ as its vertex. Half of these regions are 'positive' in the sense that they lie in $H^{+}$and correspond to regions in $H$ equipartitioning $\mu_{v}^{+}$, and the other half are 'negative'. By induction, each of these halves is once again either positive or negative. So for every sequence of $d$ elements in $\{+,-\}$ we have a unique region.

Using this we can show that $v$ is unique. Set $H_{1}=H$. Since $c$ is a Yao-Yao center, $v$ must be contained in the hyperplane $H_{2}$ through $c$ that splits $H^{+}$into 2 parts of equal measure. Note that $H_{2} \cap H$ is orthogonal to $u_{2}$ and $H_{2}$ contains a face of the region $R$ which is positive at every level. We can define hyperplanes $H_{i}$ for $i \leq d$ similarly in such a way that each $H_{i}$ is a face of $R$ and show that they are unique independently of $v$. Since $v$ is a unit vector with $v \cdot u_{1}>0$ and it is contained in $H_{2} \cap \cdots \cap H_{d}$, it has only one possible value.

Using the uniqueness of $c$ and $v$, it follows that $c$ and $v$ vary continuously with $u$.

Note that this partition has the required properties. If a hyperplane intersects the line through $c$ parallel to $v$ in $H^{+}$then it avoids one of the elements of the partition contained in $H^{-}$and vice versa.

If we want each element of the partition to have a pre-described value, then the same proof works by changing the choice of $H$ appropriately.

\section{Proof of Theorem 1}

Problems involving partitions of measures are topological in nature. There is a standard way to approach such problems, known as the test map scheme. First, we parametrize a subset of possible partitions by a space $X$ (called phase space), and 
Fig. 3 The hyperplanes, centers, and projection vectors

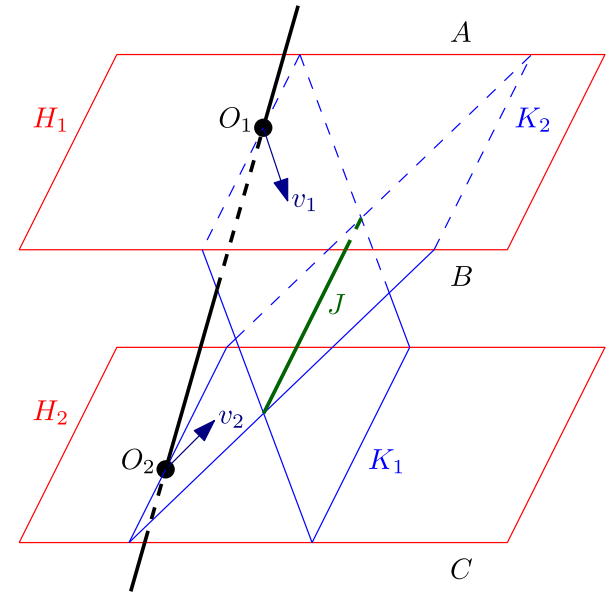

construct a space $Y$ (called target space) of parameters of a partition. These spaces are related by a natural function $f: X \rightarrow Y$ (called a test map). Ideally, there is group acting on both $X$ and $Y$ such that $f$ is equivariant. The existence of the target partition is then reduced to showing that any equivariant function on those spaces always takes some value (e.g. $[9,11])$. We follow this sketch and reduce the problem to showing that some equivariant functions always have a zero.

In our construction, we parametrize a set of partitions by $O(d)$, the space of all orthonormal basis of $\mathbb{R}^{d}$. There is an action of $\left(\mathbb{Z}_{2}\right)^{d}$ on $O(d)$, such that given $g \in$ $\left(\mathbb{Z}_{2}\right)^{d}$ and $u \in O(d), g u$ is the result of changing the sign of some elements of $u$, depending on $g$. The target space and the action on it are much more elaborate and will be shown in the proof.

We start with the geometrical part of the proof of Theorem 1 and continue with the topological part.

Let $u=\left(u_{1}, \ldots, u_{d}\right)$ be an orthonormal base of $\mathbb{R}^{d}$, we think of $u_{1}$ as the upwards direction. If $H$ is a hyperplane orthogonal to $u_{1}$, define the open half-spaces

$$
\begin{aligned}
& H^{+}=\left\{x+t u_{1}: x \in H, t>0\right\}, \\
& H^{-}=\left\{x-t u_{1}: x \in H, t>0\right\} .
\end{aligned}
$$

Let $H_{1}$ and $H_{2}$ be the hyperplanes orthogonal to $u_{1}$ such that the sets $A=H_{1}^{+}$, $B=H_{1}^{-} \cap H_{2}^{+}$and $C=H_{2}^{-}$have equal $\mu$-measure. Let $\mu_{1}=\mu \mid A \cup B$ and $\mu_{2}=$ $\mu \mid B \cup C$.

Yao-Yao's theorem applied to $\mu_{1}$ gives a unique center $O_{1} \in H_{1}$ and a unique projection vector $v_{1}$ pointing downwards (i.e. $u_{1} \cdot v_{1}<0$ ).

Let $J_{1} \subset H_{1}$ be the $(d-2)$-dimensional flat through $O_{1}$ orthogonal to $u_{2}$. Note that the hyperplane $K_{1}=\left\{J_{1}+t v_{1}: t \in \mathbb{R}\right\}$ splits $B$ into two parts of equal $\mu_{1}$-measure.

Define analogously $O_{2} \in H_{2}, v_{2}$ pointing upwards, $J_{2}$ and $K_{2}$ (see Fig. 3). Since $K_{1}$ and $K_{2}$ each divide $B$ into two parts of equal $\mu$-measure, they intersect in a $(d-2)$-dimensional flat $J \subset B$ parallel to $J_{1}$ and $J_{2}$.

The centers $O_{1}$ and $O_{2}$ as well as the vectors $v_{1}$ and $v_{2}$ vary continuously with $u$. 
Our aim is to find $u$ such that the vectors $v_{1}$ and $v_{2}$ are parallel to the line $O_{1} O_{2}$. We first show how, using this, we can construct the desired partition. Let $\mathcal{P}_{1}$ and $\mathcal{P}_{2}$ be the corresponding Yao-Yao partition to $\mu_{1}$ and $\mu_{2}$.

If this is the case, then we can use the partition $\mathcal{P}$ consisting of the elements of $\mathcal{P}_{1}$ contained in $A$, the elements of $\mathcal{P}_{2}$ contained in $C$ and the non-empty elements $K \cap B$ such that $K \in \mathcal{P}_{1}$. Let $l$ be the line that contains $O_{1}$ and $O_{2}$. Every hyperplane avoids at least two elements of $\mathcal{P}$. This is because if it hits the line $l$ in section $A$, it misses a section contained in $B$ and one contained in $C$, if it hits $l$ in $B$ it misses a section in $A$ and one in $C$ and if it hits $l$ in $C$ it misses a section in $A$ and one in $B$.

Now we use topological methods to show that there is a base $u$ satisfying the conditions above. First we need some definitions.

Given $x=\left(x_{1}, \ldots, x_{n}\right) \in \mathbb{R}^{d_{1}} \times \cdots \times \mathbb{R}^{d_{n}}$, we define $g_{i}(x)$ as the result of changing the sign of the $i$ th coordinate of $x$. We always use $g_{i}$ to denote this function independently of the target space, since it causes no confusion. We denote the $j$ th coordinate of $x_{i} \in \mathbb{R}^{d_{i}}$ by $x_{i}^{(j)}$. Given $v \in \mathbb{R}^{d-1} \times \cdots \times \mathbb{R}^{1}$, define $v^{(j)}=\left(v_{1}^{(j)}, \ldots, v_{d-j}^{(j)}\right)$ and $v^{T} \in \mathbb{R}^{d-1} \times \cdots \times \mathbb{R}^{1}$ as $v^{T}=\left(v^{(1)}, \ldots, v^{(d-1)}\right)$.

An easier way to visualize this last construction is to consider a $(d-1) \times(d-1)$ matrix $V$ induced by $v$ in the following way. In the $k$ th row write the coordinates of $v_{k}$ followed by $k-1$ signs " $\times$ ",

$$
V=\begin{aligned}
& v_{1} \\
& v_{2} \\
& v_{d-2} \\
& v_{d-1}
\end{aligned}\left(\begin{array}{ccccc}
v_{1}^{(1)} & v_{1}^{(2)} & \cdots & v_{1}^{(d-2)} & v_{1}^{(d-1)} \\
v_{2}^{(1)} & v_{2}^{(2)} & \cdots & v_{2}^{(d-2)} & \times \\
\vdots & \vdots & \ddots & \vdots & \vdots \\
v_{d-2}^{(1)} & v_{d-2}^{(2)} & \cdots & \times & \times \\
v_{d-1}^{(1)} & \times & \cdots & \times & \times
\end{array}\right) .
$$

Then $v^{T}$ is the set of vectors induced in the same way by the transpose $V^{T}$ of $V$, namely

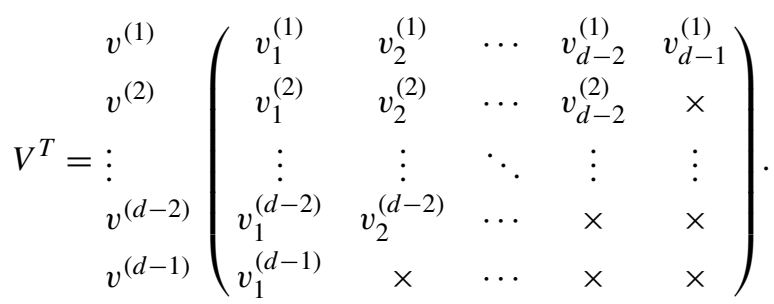

Let $r: \mathbb{R}^{d} \rightarrow \mathbb{R}^{d-1}$ be the projection such that $r\left(O_{1}\right)=r\left(O_{2}\right)=O$ and $\left\{r\left(u_{2}\right), \ldots, r\left(u_{d}\right)\right\}$ is the canonical basis.

The affine hyperplane $r(J)$ is orthogonal to $u_{2}$, so it is of the form $\left\{v: v \cdot u_{2}=\lambda\right\}$ for some $\lambda \in \mathbb{R}$. Let $x \in \mathbb{R}^{d-2}$ and $y \in \mathbb{R}^{d-2}$ be the vectors consisting of the last $d-2$ coordinates of $r\left(v_{1}\right)$ and $r\left(v_{2}\right)$, respectively.

Let $h(u)=(x, y, \lambda) \in \mathbb{R}^{d-2} \times \mathbb{R}^{d-2} \times \mathbb{R}$, note that if $h(u)=0$ for some $u$, then the vectors $v_{1}$ and $v_{2}$ are parallel to the line $O_{1} O_{2}$ and we are done. 
The map $h$ satisfies the following conditions:

- $h\left(g_{1}(u)\right)=(y, x, \lambda)$,

- $h\left(g_{2}(u)\right)=(x, y,-\lambda)$,

- $h\left(g_{i+2}(u)\right)=\left(g_{i}(x), g_{i}(y), \lambda\right)$ for $i=1, \ldots, d-3$.

Let $f: O(d) \rightarrow \mathbb{R}^{d-1} \times \mathbb{R}^{d-2} \times \cdots \times \mathbb{R}^{1}$ be defined by

$$
f(u)=((x+y, \lambda), x-y, 0, \ldots, 0) .
$$

Finding a zero of $h$ is equivalent to finding a zero of $f$. We will prove something more general.

Claim Assume $f: O(d) \rightarrow \mathbb{R}^{d-1} \times \cdots \times \mathbb{R}^{1}$ is a function such that whenever $f(u)=$ $v$, then

- $f\left(g_{1}(u)\right)=g_{2}(v)$,

- $f\left(g_{2}(u)\right)=g_{d-1}\left(v^{T}\right)^{T}$,

- $f\left(g_{i+2}(u)\right)=g_{i}\left(v^{T}\right)^{T}$ for $i=1, \ldots, d-2$.

Then there exists $u \in O(d)$ such that $f(u)=0$.

We use a similar proof method to the one Bárány used to prove the Borsuk-Ulam Theorem in [3]. This method is thoroughly explained in Chap. 2.2 of [9] and in [10].

Together with composition, we can think of $\left\{g_{1}, \ldots g_{d}\right\}$ as a set of generators of the group $\mathbb{Z}_{2}^{d}$. Given the conditions on $f$, there are natural group actions of $\mathbb{Z}_{2}^{d}$ on $O(d)$ and $\mathbb{R}^{d-1} \times \cdots \times \mathbb{R}^{1}$ such that $f$ is equivariant. However, the space $O(d)$ is too large for our needs, so instead we consider the restriction $f_{1}=f \mid S O(d)$ and the group actions of $\mathbb{Z}_{2}^{d-1}$ on $S O(d)$ and $\mathbb{R}^{d-1} \times \cdots \times \mathbb{R}^{1}$ obtained by taking the group generated by $\left\{g_{1} \circ g_{d}, \ldots, g_{d-1} \circ g_{d}\right\}$. By $g_{i} \circ g_{d}$ we mean the composition of the functions $g_{i}$ and $g_{d}$, which changes the sign of coordinates $i$ and $d$ in both the domain and the range of $f$. Since $f$ is equivariant with all the $g_{i}$, it is also equivariant with their compositions.

The main idea is of the proof is to show that, given two equivariant functions on these spaces, the parity of the number of orbits of $\mathbb{Z}_{2}^{d-1}$ in $S O(d)$ that are sent to 0 is the same. This is done via an equivariant homotopy, and it is the last step in the proof. Let us first find a function $f_{0}: S O(d) \rightarrow \mathbb{R}^{d-1} \times \ldots, \mathbb{R}^{1}$ that sends exactly one orbit of $\mathbb{Z}_{2}^{d-1}$ to 0 .

Define $f_{0}: S O(d) \rightarrow \mathbb{R}^{d-1} \times \ldots, \mathbb{R}^{1}$ as the function given by

$$
f_{0}(u)=\left(v_{1}, \ldots, v_{d-1}\right),
$$

where

- $v_{1}=\left(u_{3}^{(1)}, \ldots, u_{d}^{(1)}, u_{2}^{(1)}\right)$,

- $v_{2}=u_{1}^{(1)} \cdot\left(u_{3}^{(2)}, \ldots, u_{d}^{(2)}\right)$,

- $v_{i+2}=\left(u_{3}^{(i+2)}, \ldots, u_{d-i}^{(i+2)}\right)$ for $i=1, \ldots, d-3$.

This function is continuous. Let us show that it is also equivariant. If we show that $f_{0}$ is equivariant with all the $g_{i}$ for $1 \leq i \leq d$, then it will be equivariant with the 
subgroup generated by $g_{i} \circ g_{d}$ for $1 \leq i \leq d$. We will show each of the bullets in the conditions of $f$ hold for $f_{0}$ separately.

- If we change the sign of $u_{1}$, then only the sign of $v_{2}$ changes, so $f_{0}\left(g_{1}(u)\right)=g_{2}(v)$.

- If we change the sign of $u_{2}$, then only the last coordinate of $v_{1}$ changes. Since $v_{1}$ is the only element in the range with dimension $d-1$, this translates to $f_{0}\left(g_{2}(u)\right)=$ $g_{d-1}\left(v^{T}\right)^{T}$.

- If we change the sign of $u_{i+2}$ for $1 \leq i \leq d-2$, then we are changing the sign of the $i$ th coordinate of each $v_{j}$ (only for those $v_{j}$ that have at least $i$ coordinates). This translates to $f_{0}\left(g_{i+2}(u)\right)=g_{i}\left(v^{T}\right)^{T}$ for all $1 \leq i \leq d-2$.

Furthermore, if $f_{0}(u)=0$, then $u_{i}$ has to be the $i$ th element of the canonical basis or its negative. Therefore $f_{0}$ has exactly $2^{d-1}$ zeros in $S O(d)$. Note as well that the differential $D f_{0}$ is non-degenerate in its zeros.

Let $F: S O(d) \times I \rightarrow \mathbb{R}^{d-1} \times \cdots \times \mathbb{R}^{1}$ be the equivariant homotopy given by $F(u, t)=t f_{1}(u)+(1-t) f_{0}(u)$ that takes $f_{0}$ to $f_{1}$.

If we assume that $F$ is generic enough, then the set $F^{-1}(0)$ consists of paths and cycles. If $f_{1}$ has no zeros, then all the paths of $F^{-1}(0)$ have their endpoints at points of the form $(u, 0)$ where $u$ is a zero of $f_{0}$. Therefore there must be a path connecting two such points, but this is impossible due to the group action.

It may happen that $F$ is not generic enough. However, since $f_{0}$ has 0 as a regular value, we may perturb $F$ slightly to a map $H$ so that we have the following.

- $H$ is a homotopy between two maps $f_{0}^{\prime}$ and $f_{1}^{\prime}$, and 0 is a regular value of $H$.

- $f_{1}^{\prime}$ has no zeros.

- $f_{0}^{\prime}$ has only one orbit of points that give 0 .

Thus, we may reach a contradiction using $H$ instead of $F$.

\section{Other Proofs}

In this section we prove Proposition 2 and Theorem 3, which were stated in the introduction.

Proof of Proposition 2 We proceed inductively to construct $2 k+2$ regions $A_{1}, A_{2}$, $\ldots, A_{2 k+2}$, each of $\mu$-measure $\frac{1}{2 k+2}$, so that their boundaries are contained in the union of at most $k+1$ lines. Let $\ell_{1}$ be an oriented halving line (i.e. a line that splits $\mathbb{R}^{2}$ into two parts of equal $\mu$-measure that has fixed right and left sides). Once that $\ell_{1}, \ell_{2}, \ldots, \ell_{i-1}, \ell_{i}, A_{1}, A_{2}, \ldots, A_{i-1}$ and $A_{k+2}, A_{k+3}, \ldots, A_{k+1+(i-1)}$ have been constructed, let $\ell_{i+1}$ be the oriented halving line such that the regions

$$
\begin{gathered}
A_{i}=\left\{x \in \mathbb{R}^{2}: x \text { is right of } \ell_{i+1} \text { and left of } \ell_{i}\right\} \backslash \bigcup_{j<i} A_{j} \\
A_{k+1+i}=\left\{x \in \mathbb{R}^{2}: x \text { is left of } \ell_{i+1} \text { and right of } \ell_{i}\right\} \backslash \bigcup_{j<i} A_{k+1+j}
\end{gathered}
$$


Fig. 4 Regions for Proposition 2
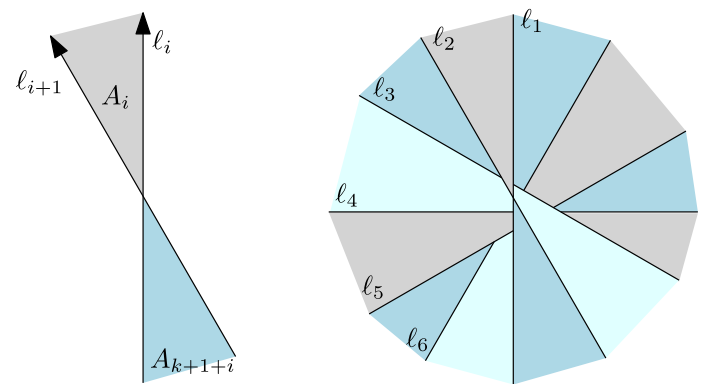

have $\mu$-measure $\frac{1}{2 k+2}$. To see that it is possible to find such $\ell_{i+1}$, consider $\mu^{\prime}$ the measure $\mu$ restricted to $\mathbb{R}^{2} \backslash \bigcup_{j<i}\left(A_{j} \cup A_{k+1+j}\right)$. Note that $\ell_{i}$ is a halving line of $\mu^{\prime}$, so we need $\ell_{i+1}$ to be a halving line of $\mu^{\prime}$ such that $\mu^{\prime}\left(A_{i}\right)=\frac{1}{2 k+2}$. This implies that $\mu^{\prime}\left(A_{k+1+i}\right)=\frac{1}{2 k+2}$. Since $\mu$ and $\mu^{\prime}$ coincide in $A_{i}$ and $A_{k+1+i}$, we obtain the desired line.

We end up with something like the right side of Fig. 4. This is a partition as $\ell_{1}$ and $\ell_{k+2}$ are equal as non-oriented lines, since at the point of constructing $\ell_{k+2}$ we have used all the measure. Since $\mathbb{R}^{2} \backslash \bigcup_{j<i}\left(A_{j} \cup A_{k+1+j}\right)$ consists of two convex components of equal measure for all $i$, every $A_{i}$ is convex. If $\ell \subset \mathbb{R}^{2}$ is a line not parallel to any $\ell_{i}$, then it intersects each of the lines $\ell_{1}, \ldots, \ell_{k+1}$ once, at every intersection $\ell$ enters at most one new region. Thus $\ell$ intersects at most $k+2$ elements of the partition. This implies that it avoids at least $k$, as we wanted.

Proof of Theorem 3 Clearly $N_{d}(k) \geq d+k$, as there is a hyperplane through any given $d$ points. The function $N_{d}$ also satisfies

$$
N_{d}\left(k_{1}+k_{2}\right) \leq N_{d}\left(k_{1}\right)+N_{d}\left(k_{2}\right) .
$$

To see this, partition $\mathbb{R}^{d}$ by a hyperplane $H$ that divides its measure in proportions $N_{d}\left(k_{1}\right): N_{d}\left(k_{2}\right)$. We can find a $k_{1}$-equipartition of one side and a $k_{2}$-equipartition of the other side. We are left with $N_{d}\left(k_{1}\right)+N_{d}\left(k_{2}\right)$ parts of equal measure such that every hyperplane avoids $k_{1}$ parts on one side of $H$ and $k_{2}$ parts in the other side of $H$.

We also have the asymptotically stronger equation

$$
N_{d}\left(k_{1} N_{d}\left(k_{2}\right)+k_{2} N_{d}\left(k_{1}\right)-k_{1} k_{2}\right) \leq N_{d}\left(k_{1}\right) N_{d}\left(k_{2}\right) \text {. }
$$

This can be shown by finding a $k_{1}$-equipartition of $\mathbb{R}^{d}$ and further partition each of its pieces by $k_{2}$-equipartitions. We are left with $N_{d}\left(k_{1}\right) N_{d}\left(k_{2}\right)$ parts of equal measure such that every hyperplane intersects at most $\left(N_{d}\left(k_{1}\right)-k_{1}\right)\left(N_{d}\left(k_{2}\right)-k_{2}\right)$ of them.

Starting with Yao-Yao's theorem and iterating (4), a sequence of partitions can be found such that in the $i$ th step we have $2^{d i}$ parts of equal measure and every hyperplane intersects at most $\left(2^{d}-1\right)^{i}$ of them. Therefore, a sequence $k_{i}$ can be found in which $N_{d}\left(k_{i}\right) / k_{i}$ tends to 1 . Then (3) implies

$$
\lim _{k \rightarrow \infty} \frac{N_{d}(k)}{k}=1
$$


Acknowledgements The authors would like to thank Imre Bárány for introducing these problems to us and for many helpful discussions. We would also like to thank the two anonymous referees for their comments and corrections, as they had a major impact on the quality of the paper.

\section{References}

1. Alon, N., Pach, J., Pinchasi, R., Radoičić, R., Sharir, M.: Crossing patterns of semi-algebraic sets. J. Comb. Theory, Ser. A 111(2), 310-326 (2005)

2. Ball, K.: An elementary introduction to modern convex geometry. In: Levy, S. (ed.) Flavors of Geometry. Mathematical Sciences Research Institute Publications, vol. 31, pp. 1-58. Cambridge Univ. Press, Cambridge (1997)

3. Bárány, I.: Borsuk's theorem through complementary pivoting. Math. Program. 18, 84-88 (1980)

4. Buck, R.C., Buck, E.F.: Equipartition of convex sets. Math. Mag. 22(4), 195-198 (1949)

5. Bukh, B., Hubard, A.: Space crossing numbers. Comb. Probab. Comput. 21(3), 358 (2012)

6. Figiel, T., Lindenstrauss, J., Milman, V.: The dimension of almost spherical sections of convex bodies. Acta Math. 139, 53-94 (1977)

7. Lehec, J.: On the Yao-Yao partition theorem. Arch. Math. 92, 366-376 (2009)

8. Lévy, P., Pellegrino, F.: Problemes concrets d'analyse fonctionnelle. Collection de monographies sur la théorie des fonctions, vol. 8. Gauthier-Villars, Paris (1951)

9. Matoušek, J.: Using the Borsuk-Ulam Theorem. Universitext. Springer, Berlin (2003)

10. Musin, O.R.: Borsuk-Ulam type theorems for manifolds. Proc. Am. Math. Soc. 140(7), 2551-2560 (2012)

11. Soberón, P.: Balanced convex partitions of measures in $\mathbb{R}^{d}$. Mathematika 58, 71-76 (2012)

12. Yao, A.C., Yao, F.F.E.: A general approach to d-dimensional geometric queries. In: Proceedings of the Seventeenth Annual ACM Symposium on Theory of Computing, pp. 163-168. ACM, New York (1985) 